of her right hand. She has clinodactyly and tongue malformations similar to the daughter. Ultrasound examination again revealed polycystic kidneys. However, renal function is normal at 44 years of age with a serum creatinine of $88 \mu \mathrm{mol} / \mathrm{l}$ and urea of $5.8 \mathrm{mmol} / \mathrm{l}$. She is of normal intelligence with no neurological dysfunction and computerised tomography of the head did not show any structural abnormality of the brain.

There is no known history of congenital malformation or of renal disease in the mother's three brothers and three sisters, in the maternal grandmother, or in the maternal great grandmother.

Chromosomal studies of both the proband and her mother have shown normal female 46,XX karyotypes on analysis of banding patterns after trypsinGiemsa staining. Both had a large heterochromatic band on chromosome 1 visible on $\mathrm{C}$ banding; such a band is usually considered to be a normal population variant.

\section{Discussion}

These two patients add to the reported cases of OFD I associated with polycystic kidneys. The proband had end stage renal failure and is only the third adult female with this combination requiring maintenance dialysis. Although a chance association with the relatively common adult type polycystic disease of the kidneys cannot be excluded, this report supports the evidence for polycystic renal changes being a distinct feature of the syndrome. Further information from more extensive screening of patients with OFD I and, when possible, necropsies, will eventually reveal the true incidence of the renal abnormality.

As many as one-third of infants with OFD I die in their first year of life and it has been suggested that neuroradiological evaluation is a valuable guide to longevity ${ }^{8}$ and prognosis. ${ }^{78}$ Agenesis of the corpus callosum in combination with cerebral and cerebellar atrophy are the likely explanations for our patient's mental retardation, communication difficulty, and clumsy gait. However, these clinical features are relatively mild and the severe structural abnormali- $-\widehat{\varnothing}$ ties of the brain in her case have been compatible with a happy life in sheltered employment.

The inheritance of OFD I is usually considered to $\vec{\circ}$ be by an $\mathrm{X}$ linked dominant gene with lethality inthe hemizygous male. The inheritance in this familyw would be compatible with this interpretation.

We thank Dr J W McNab for undertaking thí computerised tomography and Dr M J W Faed for the karyotyping.

\section{References}

1 Papillon-Léage (Mme), Psaume J. Dysmorphie des freins buccaux. Actual Odontostomatol (Paris) 1954;25:7-26.

2 Doege TC, Thuline HC, Priest JH, Norby DE, Bryant JS. Studies $\mathbb{D}$ of a family with the oral-facial-digital syndrome. $N$ Engl J Med 1964;271:1073-80.

${ }^{3}$ Tucker CC, Finley SC, Tucker ES, Finley WH. Oral-facial-digitak syndrome, with polycystic kidneys and liver: pathological andcytogenetic studies. J Med Genet 1966;3:145-7.

${ }^{4}$ Majewski F, Lenz W, Pfeiffer RA, Tunte W, Muller H. Das oro-facio-digitale Syndrom: Symptome und Prognose. Z Kinderheilkd 1972;112:89-112.

5 Harrod MJE, Stokes J, Peede LF, Goldstein JL. Polycystic kidney disease in a patient with the oral-facial-digital syndrome $\overline{0}$ type I.Clin Genet 1976;9:183-6.

6 Simon P, Tanqueral T, Toulet R, Houitte H, Ramee MP. Association de la polykystose rénale et du syndrome oral-facial-e digital. Nouv Presse Med 1978;7:122.

7 Wood BP, Young LW, Townes PL. Cerebral abnormalities in the oral-facial-digital syndrome. Pediatr Radiol 1975;3:130-6.

\& Jacquemart CJ, Trotter TL, Kaplan AM, Bcauchamp RF. The oral-facial-digital syndromes reviewed: the role of computerised axial tomography in management. Ariz Med 1980;37:261-4.

Correspondence and requests for reprints to Di W K Stewart, Department of Medicine, Ninewells Hospital and Medical School, Dundee DD1 9SY.

\title{
An oculocerebral hypopigmentation syndrome: a case report withe clinical, histochemical, and ultrastructural findings
}

\author{
M A PATTON*, M BARAITSER*, A H M HEAGERTY ${ }^{*}$, AND \\ R A J EADY† \\ ${ }^{*}$ Department of Clinical Genetics, The Hospital for Sick Children, Great Ormond Street, \\ London WC1N 3JH; and IInstitute of Dermatology, St John's Hospital for Diseases of the Skin, London E9o
}

SUMMARY A 4 year old boy is reported with tyrosinase positive hypopigmentation, mental

Received for publication 25 June 1985

Revised version accepted for publication 30 September 1985. retardation, ataxia, and myopia. Radiologica investigation showed occipital cerebral atrophy coxa valga, and generalised osteoporosis. The skin histology and electron microscopy are 
reported and discussed. The clinical features are similar to those of the oculocerebral hypopigmentation syndrome described by Preus et al. ${ }^{1}$

In 1983, Preus et al described two sibs of consanguineous Italian parents who had an oculocerebral hypopigmentation syndrome consisting of growth retardation, dolichocephaly, cataracts, high arched palate, small, widely spaced teeth, generalised hypopigmentation, psychomotor retardation, and hypochromic anaemia. This case report describes a four year old boy with a similar clinical picture. This syndrome appears to be distinct from the oculocerebral hypopigmentation syndrome described by Cross et al. ${ }^{2}$

\section{Case report}

The proband is the only child of healthy, unrelated, British parents. His mother has fair yellow hair and his father has brown hair. Pregnancy was complicated by mild hypertension. He was born at term with a birth weight of $2.65 \mathrm{~kg}$. There was no birth asphyxia. He was initially rather hypotonic and slow in feeding. At 12 weeks he was noticed to have nystagmus. His psychomotor development has been markedly delayed. He crawled at two and a half years and did not walk until three and a half years. He does not have any speech. He has had no seizures. At two and a half years he had a convergent squint corrected surgically.

He was examined at four years of age (fig 1). His height, weight, and head circumference were on the 3 rd centile. He has very fair skin and silvery white hair. His skin burns easily in the sun. He had a linear capillary naevus on the left upper arm and, in keeping with tyrosinase positive forms of hypopigmentation, there were a few pigmented naevi and pigmented hairs. His eyes and pale translucent irides and he was photophobic in moderate sunlight. $\mathrm{He}$ had a long face with a degree of ptosis. He was generally hypotonic, but without muscle weakness. He showed uncoordinated movements with a wide based, unsteady gait suggesting cerebellar ataxia. His tendon reflexes were normal. Ophthalmic examination showed a continuous pendular nystagmus. He had a full range of eye movements with an alternating convergent strabismus. He was myopic. Fundal examination under anaesthesia showed no abnormality. His electroretinogram (ERG) and visual evoked potentials (VEP) using a flash stimulus to both eyes simultaneously were normal.

The CT scan showed cerebral atrophy most marked over the occipital lobe. The skeletal survey showed a generalised osteoporosis with bilateral coxa valga and subluxation of the left hip. Other investigations showed a normal full blood count with no vacuolated lymphocytes or inclusion bodies, and amino acids, immunoglobulins, calcium, urine organic acids, TORCH screen, and chromosome karyotype were also normal.

\section{LIGHT AND ELECTRON MICROSCOPY}

Skin samples were obtained from the scalp and thigh. One part of each sample was rapidly frozen for tyrosinase studies ${ }^{3}$ and the second part was processed for transmission electron microscopy, using half strength Karnovsky medium as a primary fixative and s-collidine buffered osmium tetroxide for secondary fixation. ${ }^{4}$

Incubation of $5 \mu \mathrm{m}$ frozen sections in $0 \cdot 1 \%$ solution of L-DOPA showed a moderately strong reaction in both epidermal and hair bulb melanocytes (fig 2). The number of DOPA positive cells appeared normal. Light microscopy of semi-thin Epon sections showed that the epidermal structure

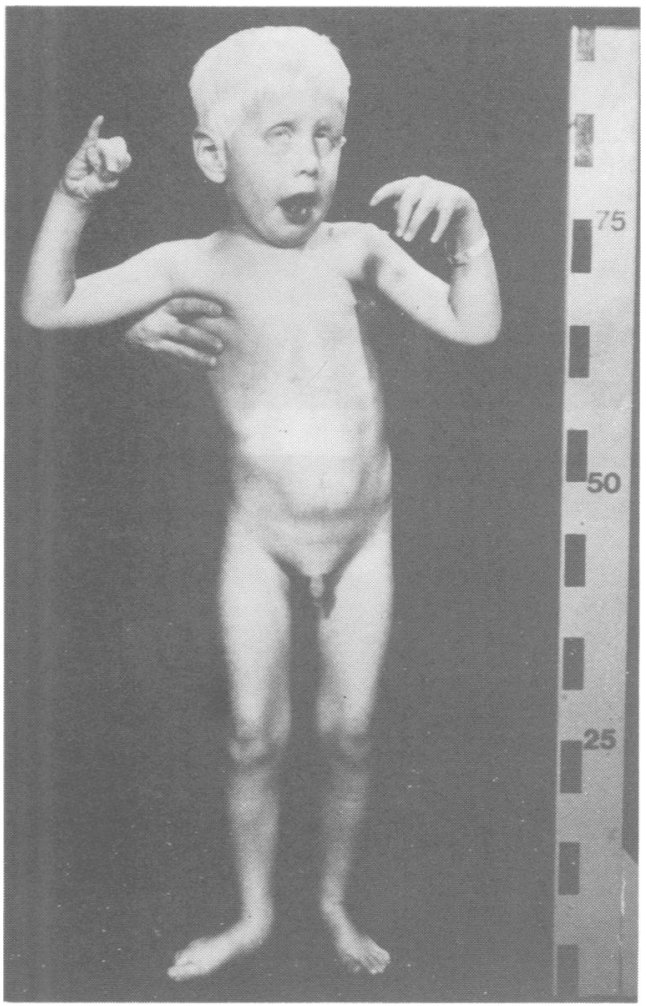

FIG 1 Patient aged four years showing silvery white hair and ptosis. 


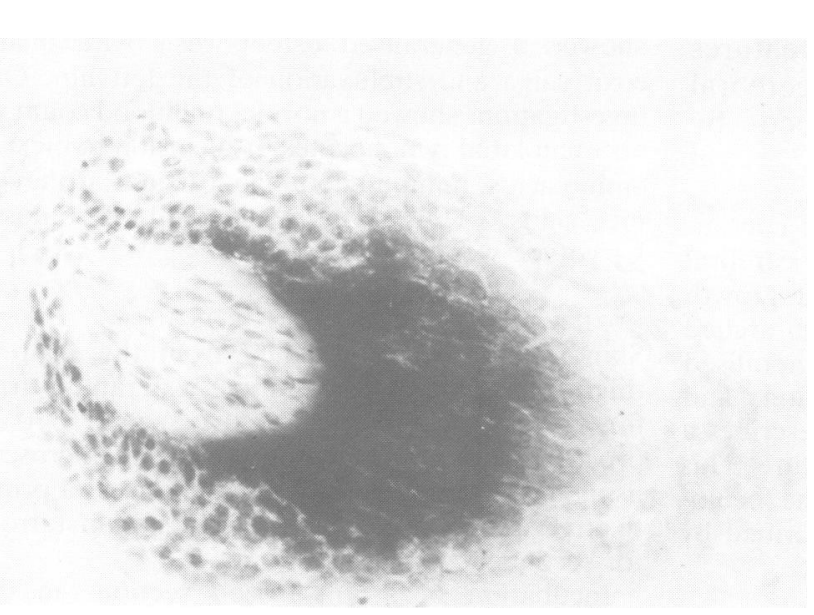

Fig 2 Photomicrograph of L-DOPA incubated cryostat section of skin from scalp.. $\overrightarrow{0}$ Note dense reaction product in hair bulb melanocytes. (Originalmagnification $\times 420$. $\overrightarrow{\mathrm{w}}$

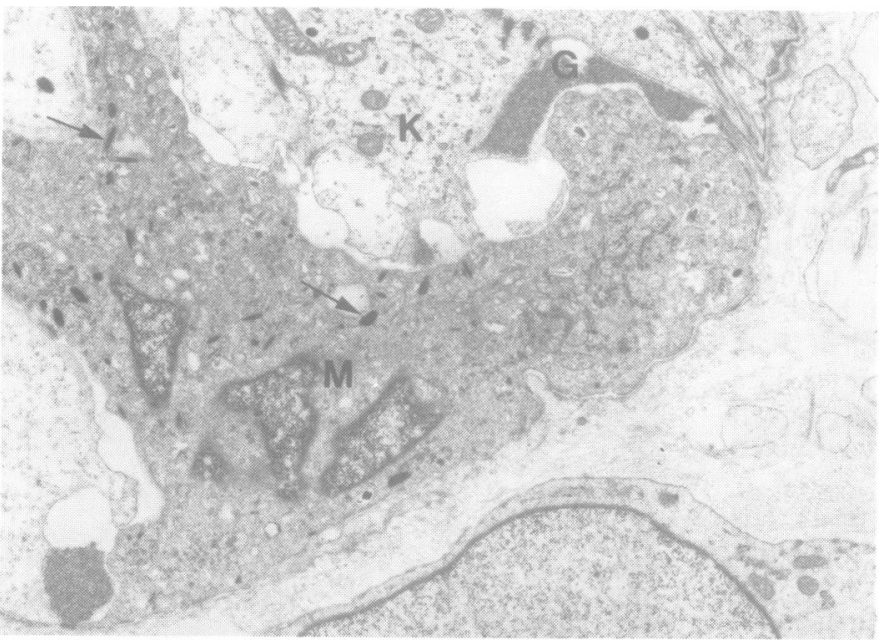

FIG 3 An epidermal melanocyte (M) containing several well melanised melanosomes (arrows) is surrounded by oedematous keratinocytes $(K)$ and intercellular granular material $(G)$. There is little evidence of melanin transfer. (Origina $\mathscr{D}_{\mathbb{Q}}$ magnification $\times 10500$.)

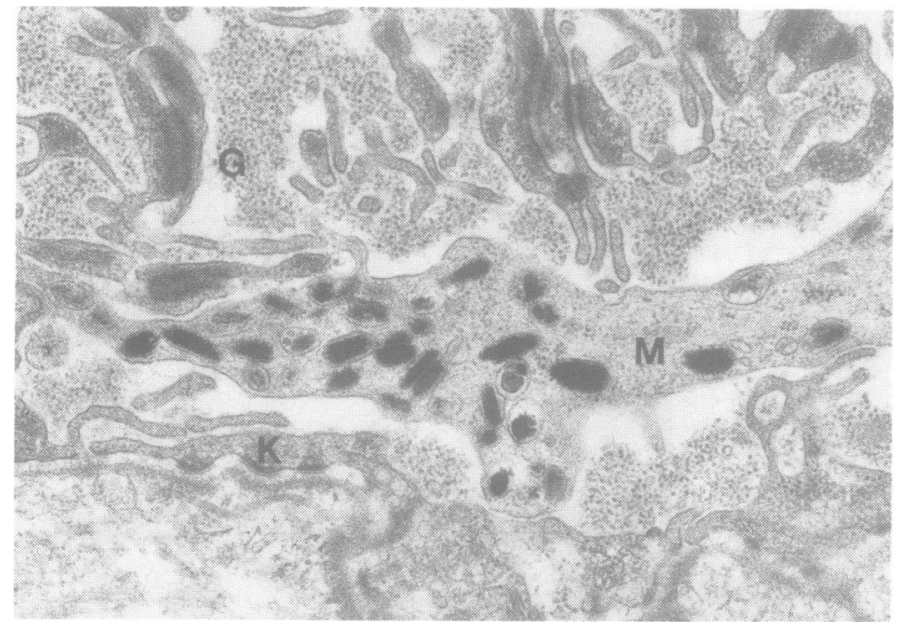

FIG 4 A melanocyte dendrite (M) contains numerous normal appearing melanosomes in different developmental stages.

Note granular material $(G)$ around melanocyte. $K=$ basal keratinocyte. (Original magnification $\times 30$ 400.)

(1)




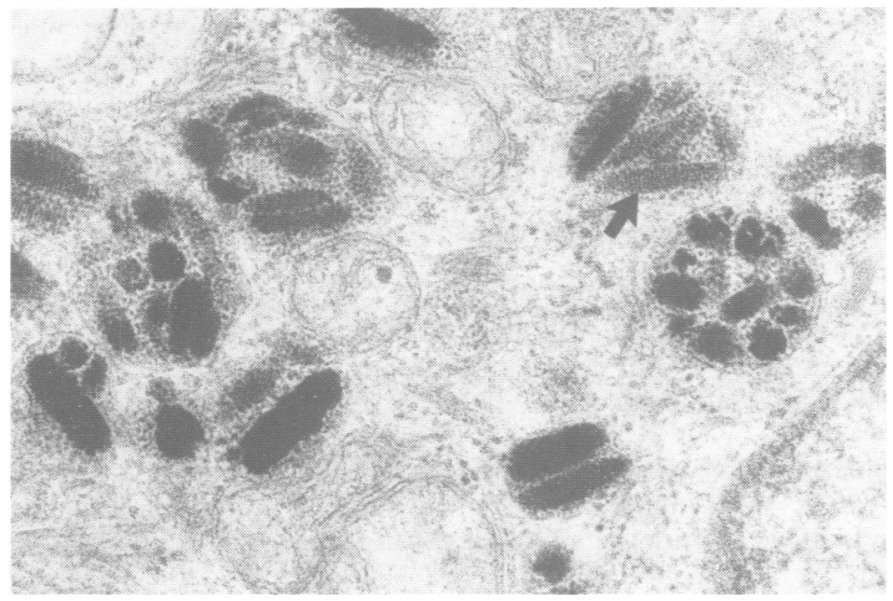

FIG 5 Melanosome complexes in basal keratinocyte. Note that some melanosomes are only in stage II (arrow). (Original magnification $\times 61000$.)

was generally normal. There was no dermal inflammatory infiltrate. Electron microscopy confirmed that epidermal melanocyte numbers were normal. The cells also appeared structurally normal and possessed dendrites.

Keratinocytes adjacent to melanocytes were often oedematous (fig 3) and suprabasal cells vacuolated, although the epidermis in general was well preserved. A granular material, which was not readily identified, was seen in the intercellular spaces of the basal layer (figs 3 and 4). At least eight melanocytes were examined in detail. All displayed evidence of active melanogenesis. There were numerous eumelanosomes up to about $0.3 \mu \mathrm{m}$ in length. Most were in stage II or III with fewer in stage IV (fig 4). Membrane bounded complexes containing two to 10 melanosomes (in stages II to IV) were seen in basal keratinocytes (fig 5) but were largely absent from the suprabasal layers. No autophagic (lysosomal) inclusions or giant melanosomes were observed within melanocytes, keratinocytes, or macrophages.

\section{Discussion}

There have been three reports of oculocerebral hypopigmentation syndromes. Cross et $a l^{2}$ reported three sibs in an inbred Amish family, who had generalised hypopigmentation with microphthalmia, corneal opacities, retardation, and spasticity. Passarge and Fuchs-Meckes ${ }^{5}$ briefly reported a three year old retarded girl, who had generalised hypopigmentation and a scaly eczema, with both spasticity and athetosis. The girl had no pupillary response to light and a flat ERG. Preus et al ${ }^{1}$ reported two sibs who had hypopigmentation, short stature, mental retardation with ataxia and decreased tendon reflexes, myopia, lens opacities, acetabular hypo- plasia, and cerebral atrophy, most marked in the occipital lobes. ${ }^{1}$

A further report by Elejalde et $a t^{6}$ described three children in a consanguineous family, who had generalised hypopigmentation and profound mental retardation, but did not have significant ocular involvement. On further investigation they were able to demonstrate abnormal melanosomes and apparently abnormal lysosomes. They therefore described the condition as neuroectodermal melanolysosomal disease.

The clinical features suggested that our patient had a hypopigmentation syndrome. The tyrosinase test showed that both epidermal and hair follicle melanocytes made substantial amounts of melanin if provided with an appropriate substrate (DOPA). There was no evidence of a structural abnormality of the melanosomal matrix. Pigment dilution in the surrounding skin was present and was almost certainly due, in the main, to the synthesis of relatively small numbers of stage IV melanosomes. A second factor causing hypopigmentation could have been the keratinocyte oedema associated with intercellular granular material, which together might have impeded melanosomal transfer. However, the possibility that these ultrastructural features were artefactual cannot be totally discounted.

Our patient and the other reported patients all had generalised hypopigmentation with severe psychomotor retardation. The ophthalmic and neurological features vary in these reports. Our patient most closely resembles the sibs described by Preus et al. ${ }^{1}$ He had cerebral atrophy with marked atrophy of the occipital lobes on the CT scan, and in the case of Preus et al a similar finding was noted at necropsy. Other features in common between the 
two reports include myopia, osteoporosis, and acetabular hypoplasia.

There is obviously some heterogeneity in this group of syndromes and the delineation of this will depend on further case reports.

It is interesting to speculate on the aetiological link between generalised hypopigmentation and mental retardation in these syndromes. Neurones and melanocytes are embryologically derived from the same origin in the neuroectoderm. It appears that melanocytes, or possibly the melanin pigments they produce, are responsible for controlling the functional development of certain neural pathways. Guillery ${ }^{7}$ has shown that the projection of the neural pathways from the retina to the visual cortex is abnormal in a number of albino mammals. This finding has been confirmed in human albinos, ${ }^{8}$ and recently it has been shown that auditory brain stem pathways in human albinos also show anomalous projections. ${ }^{9}$ It may be that this is a more generalised phenomenon leading in extreme cases to psychomotor retardation through inappropriate neural connections. In our patient there was no asymmetry in the binocular flash visual evoked potentials, but the technique used is probably insufficient to exclude the type of anomalous decussation described by Creel et al. ${ }^{8}$ Unfortunately, because of the patient's age and mental retardation, it was not felt that he would be able to cooperate in monocular pattern visual evoked potentials, which are more sensitive in delineating abnormal visual pathways. An alternative explanation is that there is a common structural or biochemical abnormality, which affects both lysosomes and melanosomes. This may be a possible explanation in the cases of Elejalde et al,${ }^{6}$ but it is unlikely in our case as he had normal melanosomes and no cytoplasmic inclusion bodies.

Mental retardation does not occur as a direct result of tyrosinase deficiency in albinos as this enzyme is not present in the central nervous system, and the conversion of tyrosine to DOPA, and hence to the dopaminergic neurotransmitters in the central nervous system, is by tyrosine hydroxylase. ${ }^{10}$
It is likely that the oculocerebral hypopigmenta tion syndromes are autosomal recessive and so in view of the severe neurological involvement au effective prenatal diagnosis would be welcome. Wל had hoped that an abnormal skin biopsy would allow prenatal diagnosis in our family, but the microscopio changes were not sufficiently distinct to allow this

We would like to thank Dr D Trounce for referring the patient, Dr P Sonksen and Dr J Wilson fo helpful discussion of the case, and Mr D B Gunne? and $\mathrm{Mr} \mathrm{B}$ Bhogal for excellent technical assistance् Dr R A J Eady is in receipt of grants from thik Rehabilitation and Medical Research Trust and the Dunhill Trust. Dr M A Patton is in receipt of a grant from the Child Health Research Trust.

\section{References}

1 Preus M, Fraser FC, Wigglesworth JW. An oculocerebr hypopigmentation syndrome. J Genet Hum 1983;31:323-8.

2 Cross HE, McKusick VA, Breen W. A new oculocerebral syndrome with hypopigmentation. J Pediatr 1967;70:398-406. Bancroft JD. An introduction to histochemical technique? London: Butterworth, 1967:230.

${ }^{4}$ Eady RAJ. Transmission electron microscopy. In: Skerrow DD Skerrow CJ, eds. Methods in skin research. London: Joh Wiley, 1985:1-36.

5 Passarge E, Fuchs-Meckes S. Oculocerebral syndrome wi hypopigmentation. Birth Defects 1975;11(2):466-7.

6 Elejalde BR, Holguin J, Valencia A, et al. Mutations affectir pigmentation in man. 1. Neuroectodermal melanolysosomal disease. Am J Med Genet 1979;3:65-80.

7 Guillery RW. Visual pathways in albinos. Sci Am 1974;230 44-54.

${ }^{8}$ Creel D, O’Donnell FF, Witkop CJ. Visual system anomalies human ocular albinos. Science 1978;201:931-3.

${ }^{9}$ Creel D, Garber SR, King RA, Witkop CJ. Auditory brainste anomalies in human albinos. Science 1980;209:1253-5.

10 Witkop CJ. Abnormalities of pigmentation. In: Emery AEHb Rimoin D, eds. Principles and practice of medical genetic Edinburgh: Churchill Livingstone, 1983:622-52.

Correspondence and requests for reprints to $D \Phi$ M A Patton, Department of Clinical Genetics, The Hospital for Sick Children, Great Ormond Stree? London WC1N 3JH. 